\title{
RF ELECTRON GUN WITH DRIVEN PLASMA CATHODE
}

\author{
I.V. Khodak, V.A. Kushnir \\ National Science Center 'Kharkov Institute of Physics \& Technology' \\ 1, Academicheskaya St., NSC KIPT, 61108 Kharkov, Ukraine
}

\begin{abstract}
The paper describes results of the experimental research of generation of intense pulsed electron beam in S-band RF gun with driven plasma cathode. There has been designed experimental sample of the cathode based on ferroelectric ceramics. At the RF gun output the generated electron beam has pulsed current of $6 \mathrm{~A}$, pulse duration of 50-90 ns and particle energy $\cong 500 \mathrm{keV}$.
\end{abstract}

\section{INTRODUCTION}

The main advantage of RF guns among electron beam sources is the ability to generate high brightness and intense beams. Generally, thermionic and photoemission cathodes are employed in RF gun for this generation. In our opinion, electron beam with pulsed current of few amperes and with pulse duration of few tens nanoseconds may be also generated by RF guns within the application of plasma cathodes based on solid dielectrics [1-3]. The source of electrons in the cathodes is the sheath of plasma which origin is a surface flashover that may be excited by driving voltage pulse. As it has been shown in numerous experimental investigations with pulsed electron DC guns [2], the emission current density of the plasma cathodes may be over $100 \mathrm{~A} / \mathrm{cm}^{2}$, and the pulse current duration can be in the nanosecond range. High peak current can be extracted from RF gun due to bunched beam structure and features of electron emission from plasma in RF field of high strength $\left(\sim 10^{7} \mathrm{~V} / \mathrm{cm}\right)$. Electrons extracted from a plasma cathode gain the RF field energy stored in the cavity of the gun. Such cathodes don't need heating and pre-activation process before operation, and may be handled and operated in vacuum of $10^{-4} \ldots 10^{-5}$ torr. Application of plasma cathodes in RF gun also potentiates the gun operation with the pulse repetition frequency of $10 \ldots 10^{2} \mathrm{~Hz}$ [3]. Results of the experimental research of S-band RF gun operation with plasma cathode are reviewed in the present paper. Plasma cathode is made from ferroelectric ceramics. The electron emission initiation on the cathode is driven by external source of pulsed voltage.

\section{EXPERIMENT APPROACH}

\section{Design Features of Plasma Cathode}

The researched plasma cathode driven by external pulse voltage was mounted in the single-cavity S-band RF gun. The base of the cathode is ferroelectric disc 2 (Fig. 1). The front side of the disc contacts with patterned electrode 1 , and the rear side is deposited by solid electrode 3 that supplies trigger pulse on the cathode.

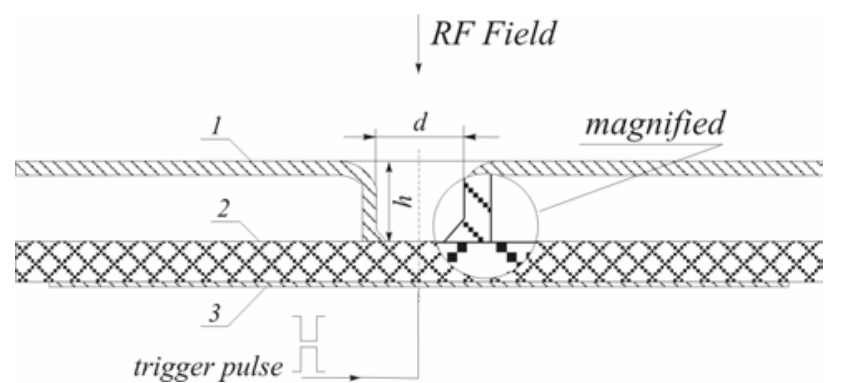

Figure 1: Cross-section of the plasma cathode.

The disc with square size of $\cong 0.8 \mathrm{~cm}^{2}$ and thickness $0.5 \mathrm{~mm}$ is made from $\mathrm{BaTiO}_{3}$ with $\varepsilon=2150$. The aperture in the patterned electrode is prolonged by cylindrical surface of finite length $h$ making cylindrical cavity. The free end face of the cavity contacts with ferroelectric disc and the opposite open plane is the interface for plasma extraction.

The driving of the surface flashover initiation on the cathode defines the principle of spatial separation of plasma development on the cathode and after-acceleration of electrons [4]. The design of RF gun plasma cathode driven by external pulse voltage was founded by the principle. The design of the cathode follows the conditions outgoing from the superposition of electrostatic field of the cathode and RF field of the gun near the cathode interface. In the first place, the electric $\mathrm{RF}$ field strength in the region of triple point junctions should be few orders of magnitude lower of the threshold magnitude of the self-excited and uncontrolled flashover [5]. In the next place, the electrostatic field of the driven voltage should not corrupt considerably the electric RF field distribution near the cathode interface that defines the initial electron dynamics.

The RF field penetrating from the gun cavity into the region of triple point junctions can be attenuated considerably by making radial $d$ and linear $h$ sizes of the cylindrical cavity in the patterned electrode too small. There was computed the axial electric RF field distribution in the interface 'cathode - gun cavity' using SUPERFISH code [6]. According to results of the computing, the attenuation of the electric RF field strength near the surface of the ferroelectric disc is $10^{4}$ times smaller for the cylindrical cavity with sizes $d=1 \mathrm{~mm}$ and $h=1 \mathrm{~mm}$ in the patterned electrode.

The end face of the cylindrical cavity contacting with the ferroelectric disc has sharped ridge (Fig. 2). This element permits to implement tangential component of electrostatic field and to induce electric field strength in the region of triple point junctions up to $\sim 10^{9} \mathrm{~V} / \mathrm{m}$ within the driving voltage of $1-3 \mathrm{kV}$. Thus, there is implemented the mode of 'strong' ferroelectric electron emission in the 
cathode [2]. It should be also noted that the ridge has an effect on the electrostatic field distribution in the interface of plasma extraction. According to the results of the computing using POISSON code [6], the ridge with sizes $0.15 \times 0.15 \mathrm{~mm}$ decreases values of tangential and radial components of electrostatic field approximately in 2 times (see Table. 1). Values $E_{\mathrm{z}}$ correspond to the axial electric field strength in the extraction interface. Values $E_{\mathrm{rmax}}$ correspond to the electric field strength at maximum radius in the extraction interface.

Table 1: Electric RF field strength in the plasma extraction interface of the cathode.

\begin{tabular}{|c|c|c|c|c|}
\hline & \multicolumn{2}{|c|}{ Without ridge } & \multicolumn{2}{c|}{ With ridge } \\
\hline$\varepsilon$ & $\begin{array}{c}E_{\mathrm{z}}, \\
\mathrm{kV} / \mathrm{cm}\end{array}$ & $\begin{array}{c}E_{\text {rmax }}, \\
\mathrm{kV} / \mathrm{cm}\end{array}$ & $\begin{array}{c}E_{\mathrm{z}}, \\
\mathrm{kV} / \mathrm{cm}\end{array}$ & $\begin{array}{c}E_{\text {rmax }}, \\
\mathrm{kV} / \mathrm{cm}\end{array}$ \\
\hline 1 & 0.51 & 0.27 & 0.32 & 0.165 \\
\hline 2000 & 1.29 & 0.67 & 0.7 & 0.36 \\
\hline
\end{tabular}

\section{Test Set-up Arrangement}

The experimental research has been carried out on the special test set-up that permits to measure parameters of electron beams with particle energy from tens of $\mathrm{keV}$ to $1 \mathrm{MeV}$. Fig. 2 illustrates the layout of main measuring equipment that has been used in the experiments.

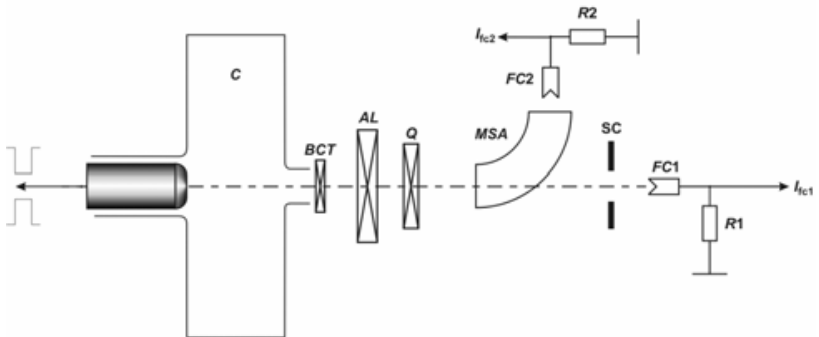

Figure 2: The layout of measuring equipment.

The cathode holder with high voltage connector supplied the cathode with a trigger pulse voltage from external pulse source. The trigger pulse has voltage up to $3 \mathrm{kV}$ amplitude, and its duration is $60 \mathrm{~ns}$. The pulsed output current having been measured by the beam current transformer $B C T$ having time resolution of $5 \mathrm{~ns}$. The magnetic lens $A L$ and quadrupole $Q$ supplied beam transport. Electron energy of the beam having been measured using magnetic analyzer MSA and Faraday cup $F C 2$. The beam profile having been measured by driving slits $S C$ and Faraday cup $F C 1$. The gun was fed by klystron RF amplifier operating in self-excited mode with operating frequency of $2797.15 \mathrm{MHz}$. The pulse RF power $P_{c}$ feeding the gun is in the range $0.1 \ldots 1 \mathrm{MW}$ with pulse duration $1.8 \mu \mathrm{s}$. The axial electric field strength in the RF gun is determined by the following expression evaluated from resonant perturbation measurements of the field:

$$
E(V / m)=470 \sqrt{P_{c}(W) Q_{0}},
$$

where $Q_{0}$ is unloaded quality factor of the gun cavity.

\section{RESULTS OF THE RESEARCH}

There has been the purpose of the research of RF gun operation with plasma cathode to measure parameters of the generated beam current and the identification of featured beam current dependences on electric RF field strength and on driving voltage.

The cathode was fed by trigger pulses $U_{\text {tr }}$ both positive and negative polarity during the research. The patterned electrode of the cathode was grounded. Results of measurements of generated beam current has shown up the time difference in the beginning of the current pulse rise at RF gun output and in the duration of this pulse for different polarity of $U_{\mathrm{tr}}$. For the negative polarity of $U_{\mathrm{tr}}$ the peak amplitude of the gun output beam current has time delay $\cong 50 \mathrm{~ns}$ after the pulse voltage $U_{\text {tr }}$ falls to zero value. The duration of the beam current pulse is $50 \mathrm{~ns}$ in this case. For the positive polarity of $U_{\text {tr }}$ the gun output current runs into the peak value just after the pulse voltage $U_{\text {tr }}$ falls to zero value. The duration of the beam current pulse is $90 \mathrm{~ns}$ in this case.

The one of interpretations of the observed difference is the confinement of plasma bulk in the cylindrical cavity of the patterned electrode under the negative pulse of $U_{\text {tr }}$. It should be also noted that peak value of the beam current pulse is of the same value both for negative and positive polarity of the pulse $U_{\text {tr }}$.

Results of measurements of the dependence of the output beam current on average electric field strength in the gun cavity are shown on the Fig. 3 .

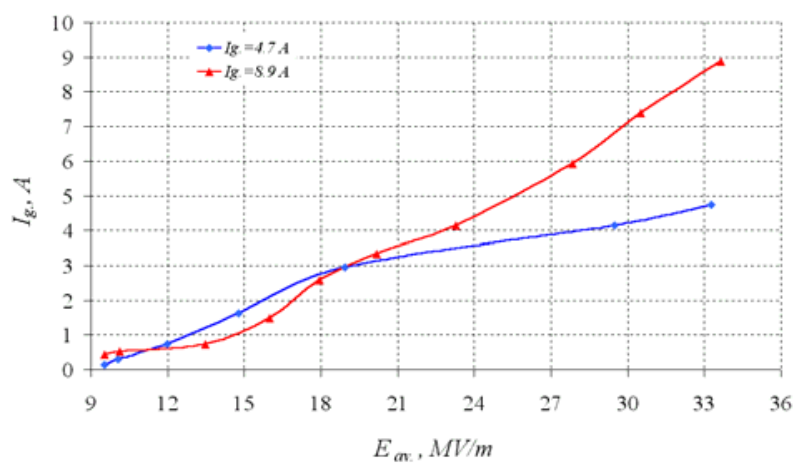

Figure 3: Beam current dependences.

The dependences have been measured for two different maximum values of the gun output current that were set by the voltage value of the pulse $U_{\text {tr. }}$. As one can see from the dependences, the mode of RF gun operation with pulse current $4.7 \mathrm{~A}$ is close to the mode of space charge saturation similar to DC guns. Comparative analysis of measured results with results of numerical simulation of particle dynamics in the beam using PARMELA code [7] has shown that emission current density is approximately $\approx 9 \cdot 10^{2} \mathrm{~A} / \mathrm{cm}^{2}$ in this case (Fig. 4). The particle energy estimated after the results of the analysis is $\cong 500 \mathrm{keV}$ that fits the experimental investigation of RF gun operation with metal-dielectric cathodes [8]. 


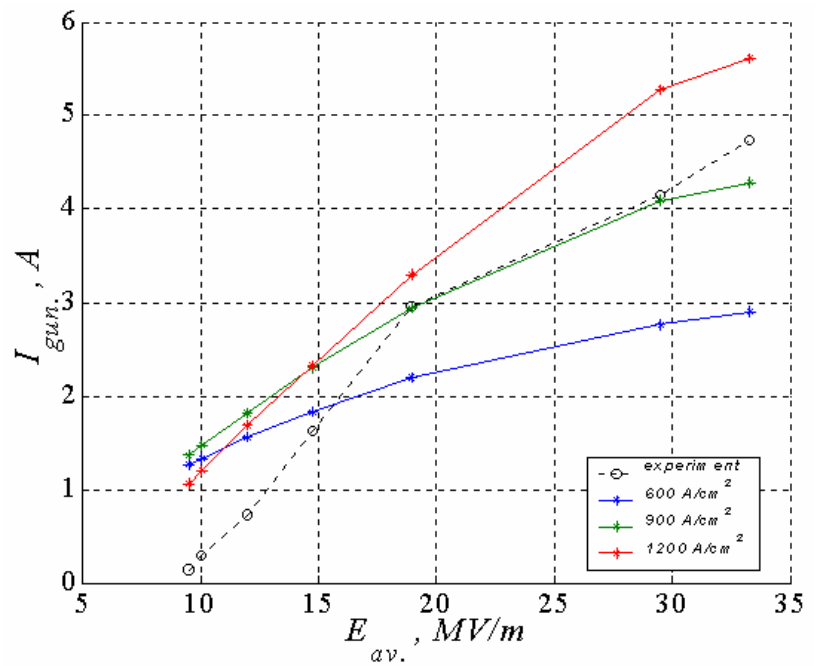

Figure 4: Results of comparative analysis.

The dependence of maximum pulse current of the gun on the average electric field strength in the gun cavity goes like linear function (Fig. 3) for the gun output current more than $4.7 \mathrm{~A}$. This fact permits to assume that there is no saturation of the current transmitted to the gun output under the increasing of the current extracted from plasma within the same range of average electric field strength values. According to the results of the analysis, the saturation of the transmitted current occurs for the average electric field strength more than $35 \mathrm{MV} / \mathrm{m}$, and the gun output current is limited by the particle loss in the gun cavity.

There has been measured the profile of the generated electron beam in two transverse planes during the research. Fig. 6 demonstrates the plane projection of the two-dimensional beam current distribution. The dotted line corresponds to the FWHM level of the distribution. According to results of profile measurements, the beam generated by RF gun with plasma cathode may considered as axially symmetric with $6.5 \mathrm{~mm}$ diameter.

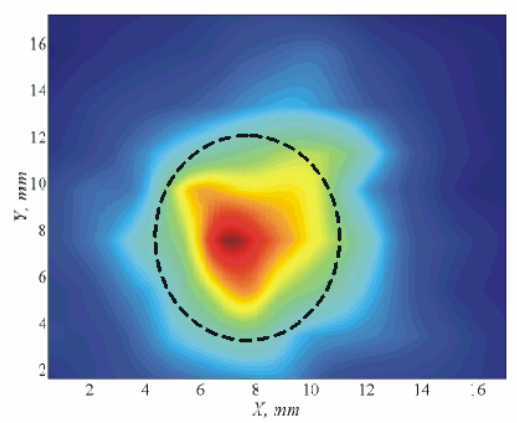

Figure 5: Measured beam profile.

\section{CONCLUSION}

It was established in the research that RF guns with plasma cathodes based on ferroelectric ceramics can generate pulse electron beams with pulse current up to
$10 \mathrm{~A}$ and with pulse duration few tens nanoseconds. It should be noted that within the phase length of electron bunch in RF gun of $\Delta \varphi$ the peak current value in the bunch is $I_{\mathrm{b}}=I_{\mathrm{p}} \cdot(2 \cdot \pi) / \Delta \varphi$. It means that for a bunch phase length about 0.7 rad the peak current in a bunch at RF gun output may be up to $10^{2}$ A with particle energy $\cong 500 \mathrm{keV}$.

\section{ACKNOWLEDGEMENT}

The authors are exceedingly grateful to the staff of R\&D "Accelerator" of NSC KIPT for their help throughout the experiment period. Special thanks are to Prof. A.N. Dovbnya for the support of the work, to Dr. V.V. Mitrochenko and Dr. V.F. Zhiglo for useful discussions of the results, to Dr. V.V. Zakutin and V.P. Romas'ko for the design of some experimental equipment.

\section{REFERENCES}

[1] V.S. Balagura， B.G. Safronov， S.A. Cherenshchikov Short-pulse electron guns with unheated cathodes for linacs, Problems of Atomic Science and Technology. Ser.: Nuclear-physics research (theory and experiment), №4(25), (1992), p. 48-51. (in Russian)

[2] G. Rosenman, D. Shur, Ya. E. Krasik and A. Dunaevsky, Electron emission from ferroelectrics, J. Appl. Phys. 2000, v. 88, p. 6109-6161.

[3] N.I. Aizatskii, E.Z. Biller, I.V. Khodak et al. Metalinsulator cathode in an $\mathrm{rf}$ electron gun, Technical Physics Letters (An English translation of Russian journal "Pis'ma v Zhurnal Tekhnicheskoi Fiziki"). 1998 , v. 24, № 10, p. 762-763.

[4] S.V. Bulychyov, A.E. Dubinov, V.S. Zhdanov et al., "Generation of Wide-Band Radio-Pulses by Reflex Triode with Virtual Cathode", Proc. of III Scientific Seminar in Memory of V.P. Sarantsev, Dubna, Russia, 1999, P.68-75 (in Russian).

[5] G. A. Mesyatz， D. I. Proskurovskiy， Pulse electric discharge in vacuum, Novosibirsk: Nauka, 1984, 256 p. (in Russian)

[6] J.H. Billen and L.M. Young "POISSON/SUPERFISH on PC compatibles", PAC'93, Washington, 1993, p.790.

[7] L.M. Young, "PARMELA", Los Alamos National Laboratory, LA-UR-96-1835 (preprint), Los Alamos, 1996, 93 p.

[8] I.V. Khodak, V.A. Kushnir, "Performances of the beam generated by metal-dielectric cathodes in RF electron guns", EPAC'04, Lucerne Switzerland, P.767-769. 